\title{
$F$-center mechanism of long-term relaxation in lead zirconate-titanate-based piezoelectric ceramics. 1. After-heating relaxation
}

\author{
V. M. Ishchuk* and D. V. Kuzenko \\ Science \& Technology Center "Reaktivelektron" \\ of the National Academy Science of Ukraine \\ Bakinskikh Komissarov Street, 83049 Donetsk, Ukraine \\ *valerii.ishchuk@gmail.com
}

Received 2 September 2015; Revised 23 November 2015; Accepted 23 November 2015; Published 31 December 2015

\begin{abstract}
Results of experimental investigation of relaxation aging processes in $\mathrm{Pb}(\mathrm{Zr}, \mathrm{Ti}) \mathrm{O}_{3}$-based solid solutions after termination of external actions are presented. Heating, DC electric field, uniaxial pressure and some of their combinations were taken as external actions. In the main part of the present paper, we use heating as external action. The said processes are long-time one and are described by the logarithmic function of time.

Reversible and nonreversible relaxation processes take place depending on the action intensity. The relaxation rate depends on the action intensity also, and the said dependence has nonlinear and nonmonotonic form if external action leads to domain disordering.

The oxygen vacancies-based model for description of the long-time relaxation processes is suggested. The model takes into account oxygen vacancies on the sample's surface ends, their conversion into $F^{+}$- and $F^{0}$-centers under external effects (due to the liberation of the pyroelectric charge) and subsequent relaxation of these centers into the simple oxygen vacancies after the actions termination. The initial sample's state is electroneutrality one. F-center formation leads to the violation of the original sample's electroneutrality, and generates DC electric field into the sample. Relaxation of $F$-centers is accompanied by decreasing of electric field, induced by them, and dielectric constant relaxation as consequent effect.
\end{abstract}

Keywords: Piezoelectric; after-heating process; long-term relaxation; dielectric constant; $F$-center.

\section{Introduction}

Piezoelectric ceramics on the base of lead zirconate-titanate (PZT) find wide practical applications as sensors, electromechanical devices, transformers and converters, ultrasound sources. ${ }^{1,2}$ Now different kinds of sensors are used under the hardening of requirements to safe work of devices with moving working part in industry and in all kinds of transport. ${ }^{3,4}$ Large part of the said sensors operates under "startstop" conditions. There are many investigations considering the behavior of piezoceramic elements under the effect of external factors such as electric field, temperature and mechanical stress. But relaxation properties of materials after the removal of external physical actions have been studied insufficiently till this date. ${ }^{5-9}$

The aim of the present paper is to study the properties of PZT-based piezoelectric ceramics after the termination of the action of external thermodynamic parameters such as DC electric field, temperature, uniaxial stress and their combinations. We pay main attention to sample heating for external effect.

We have investigated both cases when external actions lead and do not lead to irreversible processes. All relaxation processes have been studied for a long time.

\section{Experimental Methods}

The disk-shaped piezoceramic elements of standard size $d=10 \mathrm{~mm}$ and $h=1 \mathrm{~mm}$ were used in the present study. The elements were obtained from the PZT-based powders. The investigated PZT solid solutions locate in the "composition $(\mathrm{Zr} / \mathrm{Ti})$ - temperature" diagram of phase states both in the regions of rhombohedral $(R h)$ and tetragonal $(T)$ distortions of the perovskite crystal lattice as well as in the morphotropic region. The latter is characterized by the coexistence of domains or $R h$ - and $T$-phases in the sample's volume.

Ceramics samples were obtained by the traditional ceramic technology via the two-stage sintering at $850^{\circ} \mathrm{C}$ and $1200^{\circ} \mathrm{C}$. Single phase nature of ceramics was controlled by the X-ray diffractometer IIPOH-3 (X-ray diffractometer for general mission) using the filtered $\mathrm{CuK}_{\alpha}$ emission. The residual porosity of samples was not more than $0.2 \%$. After sintering and polishing the diameter and thickness of ceramic samples were $10 \mathrm{~mm}$ and $1 \mathrm{~mm}$, correspondingly.

Silver electrodes were used for dielectric and piezoelectric measurements. The polarization of ceramic samples was carried out at a temperature $120^{\circ} \mathrm{C}$ in $\mathrm{DC}$ electric field with intensity $3 \mathrm{kV} / \mathrm{mm}$ during $1 \mathrm{~h}$ with the subsequent cooling down to room temperature in the field.

This is an Open Access article published by World Scientific Publishing Company. It is distributed under the terms of the Creative Commons Attribution 4.0 (CC-BY) License. Further distribution of this work is permitted, provided the original work is properly cited. 
As the external ambient actions, which turn out the sample from the state of thermodynamic equilibrium in our experiments were heating of samples, uniaxial mechanical stress and DC electric field directed along and against to the polarization direction. Before the beginning of the each experiment, samples were kept for at least one month under normal conditions.

Heating of the sample was conducted in the low-inertia electric furnace with the chromel-alumel thermocouple. The heating temperature was varied from $80^{\circ} \mathrm{C}$ to $350^{\circ} \mathrm{C}$, the time of endurance was $10 \mathrm{~min}$. Isothermal endurance was carried out with precision $\pm 0.75^{\circ} \mathrm{C}$. After that procedure, the sample was rapid cooled (quenched) to room temperature.

Mechanical uniaxial pressure was created with the spring press with precision $\pm 2 \%$. Holding time under the load was $10 \mathrm{~min}$. Each of the heating and uniaxial loading of PCE was made in the electrical short-circuited state.

Temperature dependencies of the sample's permittivity (dielectric constant $\varepsilon$ ) were carried out in AC electric field with intensity $0.1 \mathrm{~V} / \mathrm{mm}$ and frequency $1 \mathrm{kHz}$ using precision RLC-meter E 7-20. Temperature dependencies of remnant polarization were obtained by integration of electric charge released under heating and thermal depolarization of the samples.

The aged procedure after each physical action switching off was carried out at room temperature $\left(22^{\circ} \mathrm{C}\right)$ during 30 $40 \mathrm{~h}$ and dielectric constant was measured during all this time.

\section{Reversible Relaxation}

\subsection{Experimental results}

When working with ferroelectrics, the question always arises of what parameters should be monitored. At first look, the answer is straightforward - it is the polarization that is the order parameter. But unlike a magnetization into magnetic substances, in ferroelectrics a polarization is not amenable to direct measurement. All information about its behavior can be obtained only indirectly - for example, by the calculations based on the structural data. But everything in this direction are model calculations, and the final result depends on the choice of the model. As practice shows, such calculations give more or less acceptable results only for simple compounds. PZT-based solid solutions are not such.

The most appropriate physical characteristic, that is available for observation and carries information about the order parameter, is the dielectric constant measured in weak fields ( $\varepsilon=d P / d E$ ). Its measurement at each time takes $1-2 \mathrm{~s}$, so the behavior of dielectric constant allows to follow the relaxation processes in the early stages. Not the last argument in favor of using the dielectric constant is the large accuracy of the measurement of its value.

For all considered cases of the external actions the relaxation of dielectric constant $\varepsilon$ after the termination of action

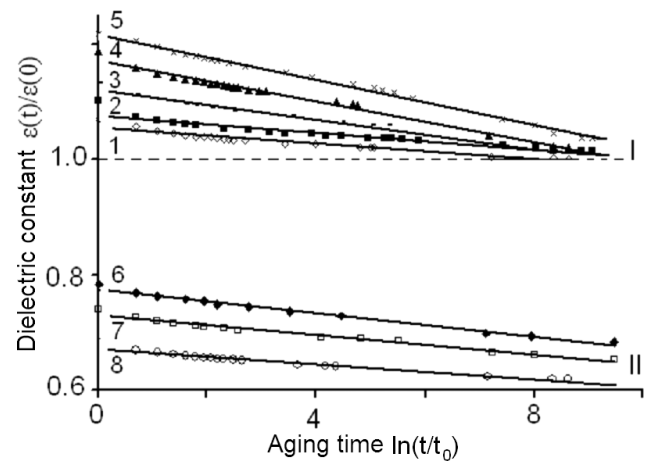

Fig. 1. Relaxation dependences of the relative dielectric constant for $\left(\mathrm{Pb}_{0.95} \mathrm{Sr}_{0.05}\right)\left(\mathrm{Zr}_{0.53} \mathrm{Ti}_{0.47}\right) \mathrm{O}_{3}$ solid solution after heating in the interval of reversible (I) and irreversible (II) dielectric constant variation $\left(t_{0}=1 \mathrm{~min}\right)$. More about intervals I and II see in Sec. 4.1. Temperature interval, ${ }^{\circ} \mathrm{C}$ : I, $100-240\left(T<T_{d}\right)$, II, 260-290 $\left(T_{d}<T<T_{C}\right) . \mathrm{T},{ }^{\circ} \mathrm{C}: 1-100,2-130,3-187,4-214,5-$ $240,6-280,7-289,8-290$.

is described by logarithmic time function

$$
\varepsilon(t) / \varepsilon_{0}=-A \times \ln \left(t / t_{0}\right)+B,
$$

where $t$ is aging time after removal of external action, $\varepsilon(t)$ is the dielectric constant measured at the moment of time $t$ and $t$ is aging time, $\varepsilon_{0}$ is the dielectric constant measured before external action. As an example, Fig. 1 shows the time dependences of dielectric constant after heating termination and flash cooling to room temperature.

The factor of reliability of approximation $R^{2}$ (defined by MS Excel) for all heating temperatures is in the interval from 0.9805 to 0.9947 . Such values of $R^{2}$ (close to 1.0000 ) point out the high accuracy of the linear approximation of experimental results and subsequent obtaining of the coefficients $A$ and $B$. Owing to high accuracy of permeability measurements, close to 1.0000 of $R^{2}$ values and the big array of experimental points, coefficients $A$ and $B$ errors are small (relative error not exceed $0.96 \%$ for $A$-obtaining and $1.17 \%$ for $B$-obtaining) and cannot be shown on the dependences of these coefficients on experiment parameters which will be considered further in the paper (Figs. 2, 5, 8 and 9).

The expression (1) can be rewritten in the form which is adopted in the study of relaxation processes:

$$
\varepsilon(t) / \varepsilon_{0}=-A \times \ln (t / \tau),
$$

where $\tau=\exp (B / A)$. The presence of the logarithmic dependence in Eqs. (1) and (2) points to the wide range of relaxation times. ${ }^{10,11}$ These equations are used for studying of ferroelectric ceramics as well as ferrites.

For all external effects investigated by us, in the case of low action intensity there is a reversible relaxation - values of measured parameters come back to the initial values during the aging (temperature interval I in Fig. 1 as example of the reversible after-thermal relaxation). As will be shown below, small loads are those in which there is no domain disordering. 

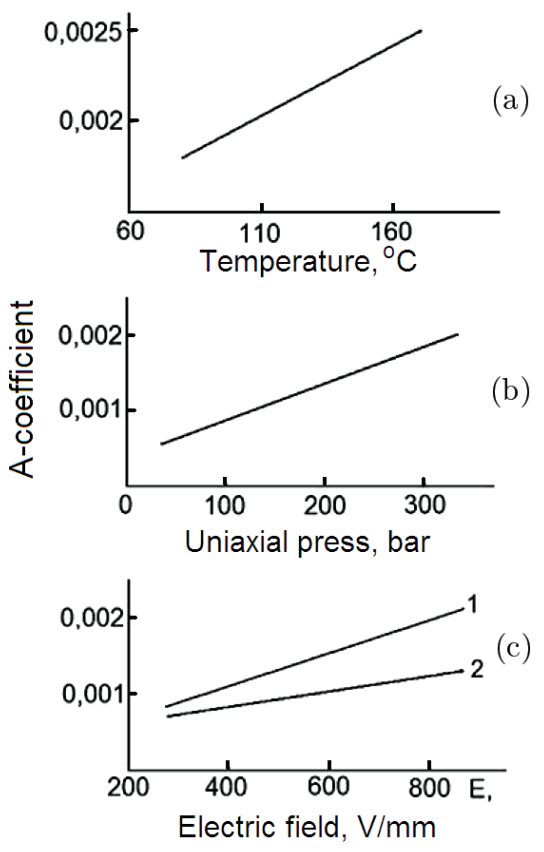

Fig. 2. Dependences of $A$-coefficients on amplitude of physical actions for solid solution $\left(\mathrm{Pb}_{0.95} \mathrm{Sr}_{0.05}\right)\left(\mathrm{Zr}_{0.53} \mathrm{Ti}_{0.47}\right) \mathrm{O}_{3}$. Type of excitation: (a) heating; (b) uniaxial mechanical compression; (c) DC electric field with field direction against (1) and along (2) to polarization of the sample.

Dependences of $A$-coefficient, characterized by the relaxation rate, on the intensity of external actions of different physical nature are presented in Fig. 2 for solid solution $\left(\mathrm{Pb}_{0.95} \mathrm{Sr}_{0.05}\right)\left(\mathrm{Zr}_{0.53} \mathrm{Ti}_{0.47}\right) \mathrm{O}_{3}$. Part of the experimental results relating to the low intensity influence, was partially published in the Russian press. ${ }^{12}$ The relaxation rate linearly increases with increasing of the effect intensity for all cases. We named such relaxation as linear one.

While solid solutions from $R h, T$ or morphotropic region of PZT phase diagram were investigated, qualitative (and considerable quantitative) distinctions have not been observed.

\subsection{Hypothesis about the nature of after-effect relaxation}

The results obtained in the study of the so-called linear relaxation put some questions, the answers to which can help in the search of mechanisms of long-term after-effect relaxation and for building of the model of the studied processes.

The first that we must emphasize is related to the time of the external effects and after-effects. Each action in itself lasts a few minutes while the after-effect process stretched for tens of hours. As far as is known from the literature, there is no answer to this question to date.

The next important peculiarity of the studied relaxation processes is associated with unidirectional change in the dielectric constant - during relaxation it only decreases and never rises. Such behavior seems quite natural after a temperature or pressure effect (both of these effects lead to the dielectric constant increase). But when external DC electric field is directed along the sample's polarization the dielectric constant decreases. It would seem that after field switchingoff dielectric constant should increase. But it goes down. How come? To this question in the literature there is no answer.

Experimental results in this section show the relaxation law (1) is carried out at the aging of samples in all cases not depending on the physical nature of external action. The relaxation rate almost does not depend on the physical nature of external action. Therefore, we can draw a conclusion that identical physical processes of relaxation take place after external actions of different nature on samples in the case of low action intensity. The unique common mechanism determines the after-effect relaxation.

All three of the above-mentioned features of long-term relaxation allow us to put forward the hypothesis about it's physical mechanism, which has been tested in the distance of further investigations.

In all above examples, the external actions cause a partial depolarization of the sample. As a consequence, a surface electric charge, uncompensated by the polarization field (pyrocharge), is released on the sample's surface ends. At the cancellation of the external actions, the remnant polarization is returned to its original state and electric charge released earlier is bound by the restored sample polarization.

Together with the above process the electric charge is released on the sample's surfaces under external actions which is captured by crystal lattice defects. In the PZT solid solutions, such lattice defects are predominantly oxygen vacancies in the anion sublattice and lead vacancies in the $A$ sublattice of the perovskite crystal structure. ${ }^{13-16}$ Since actual charge carriers are negatively charged electrons, the focus should be given to their capture by positively charged oxygen vacancies.

As for the case of the electric field applied in the direction of polarization, in this case the excess charge is generated by a voltage source. After field switching-off all processes are analogous to ones that were considered above.

As a result, after electrons capture by oxygen vacancies, $F^{+}$- or $F^{0}$-centers are formed. The defects under discussion are concentrated on the sample's surface ends (for polarized sample) and, to a lesser extent, on heterogeneities of sample. ${ }^{13,17-19}$ As example, grain boundaries can be such heterogeneities. In depolarized sample the said defects are concentrated on the domain surface ends.

Excited $F$-centers are separated from the ground-state (net oxygen vacancy) by a potential barrier. ${ }^{14,20}$ Potential barrier height is from $0.3 \mathrm{eV}$ to $1.5 \mathrm{eV}$ for different oxides. ${ }^{14,19,21,22}$ In oxides with the perovskite crystal structure, this interval is somewhat narrower. After the sample quenching, returning of the remnant polarization to the initial state and binding of the charge released before the relaxation of $F^{+}$- and $F^{0}$-centers to the ground state begins. For the above said heights of potential barriers, the time required to restitution of the 
original state ranges from several tens to several hundreds of hours. There is these relaxation times we observed after external effects of different nature on the sample.

Before external effects, piezoelectric element was in the electrical neutral state. After the $F$-center creation, the initial neutral state is disturbed - now $F$-center represents itself as charged defects. After external influence termination, excess charge of $F$-centers remains on the face ends for a long time. It creates an electric field, which leads to a change in the dielectric constant (as a result, to a change in the resonant frequency also). This field exists throughout the entire period of time while charged $F$-centers exist. As far as the $F$-centers decay, the induced electric field tends to zero and the dielectric constant tends to its initial value $\varepsilon_{0}$.

This discussed hypothesis gives answers to the questions that before our work had no answers.

Firstly, there is a significant difference in the times of external effects and after-effect relaxation. During external effects, there is liberation of electrons that are trapped by oxygen vacancies. For electron capture by oxygen vacancies with the $F$-center formation (and as a consequence, with the creation of an additional electric field varying dielectric constant), the scale of the times is virtually instantaneous in comparison with one we operate in experiments. The subsequent decay of the charge defects occur for a long time (tens of hours), and a long-time relaxation is observed. Therefore, our hypothesis explains large differences of the duration of external effect and the time of relaxation.

Secondly, in all cases after removal of the external effect, there is a decrease, in sample's permittivity only. To understand why this is happening, we refer to Fig. 3, which shows the loop of electric hysteresis for dielectric constant. Prior to external influences on the sample it is in the initial state, which corresponds to point 0 on the hysteresis curve. The liberation of electric charge at the partial depolarization of the polarized sample (under external action of any physical feature) leads to the appearance of some electric field with the direction opposite to the polarization one. The passage on the hysteresis loop from point 0 to point 1 takes place. After termination of external action, most of the released charge is

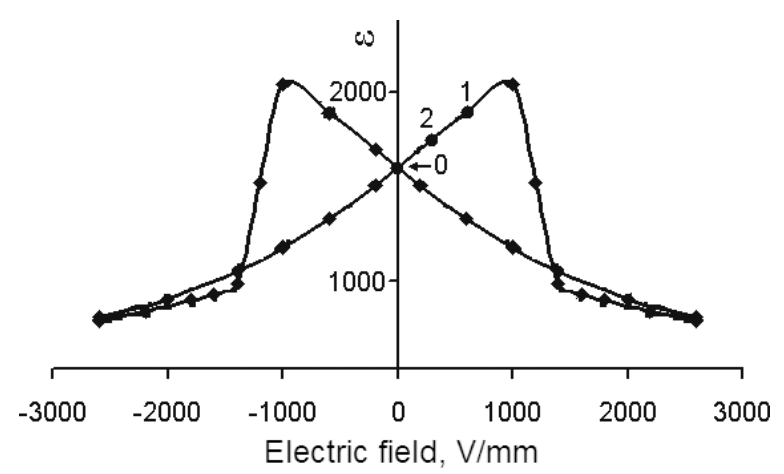

Fig. 3. Hysteresis loop for dielectric constant of nonpolarized PZT 52/48 solid solution. coupled by restored polarization, the field produced by this charge is reduced and there is a rapid passage from point 1 to point 2.

Due to the fact that part of the charge is associated by crystal lattice vacancies (with formation of $F$-centers located on the end surfaces of the sample), the sample is exposed to "external to him" electric field. Therefore, the dielectric constant is higher than it's initial value. During the relaxation process, $F$-centers return into the initial state $\left(\mathrm{O}^{2+}\right.$-vacancies). The field created by them goes to zero, and dielectric constant returns to the initial value (passage from point 2 to point 0 ). For this reason, dielectric constant decreases only in the process of the after-effect relaxation.

The following sections of this article comprise a material that confirms our hypothesis and allows us to draw a conclusion that it overgrew into a model of long-term after-effect relaxation. But already now we give a result that seriously speaks in favor of this.

If all effects that we have considered so far are caused by pyrocharge, in the absence of sample polarization such effects should not exist - there should be no relaxation of dielectric constant. To test this conclusion, the samples must be identical in all aspects to those on which we have conducted experiments, but they should not have the spontaneous and remnant polarization. For most materials, such samples cannot choose, but it is not true for PZT-based solid solutions. For PZT system at small content of titanium, the low-temperature state (at temperatures below the Curie point $T_{C}=230^{\circ} \mathrm{C}$ ) of solid solutions is antiferroelectric (AFE) and spontaneous polarization is absent. In all other aspects, these solid solutions are identical to those that are placed in the region of ferroelectric (FE) state of the phase diagram and show after-effect relaxation: they are obtained in the same technology, they have the same sintering temperatures, they have similar values of the lattice parameters, they have the same microstructure of the sintered ceramic samples, and the structure of lattice defects is the same as in ferroelectric solid solutions.

Not all types of external effects are suitable for checking our hypothesis about feature of relaxation. We should immediately leave aside any action using an electric field, because external voltage source will always provide "recharge" of oxygen vacancies. Thermal action is also not suitable. Despite the fact that the region of AFE states in PZT phase diagram is shown usually as a single phase, in fact, such statement is true only for "average" review. The results of precision X-ray and neutron-ray studies (for example, see Ref. 23 and references therein) as well as Raman scattering ${ }^{24}$ show, even in the pure lead zirconate, local domains (in the scale up to 10 lattice constant) undergo crystal lattice distortions and may have a dipole moment (of course, on a macroscopic scale lead zirconate at temperatures $T<T_{c}$ is antiferroelectrics). All of these lead to abandon thermal action to checking $F$-center hypothesis when working with PZTbased AFE solid solutions. 


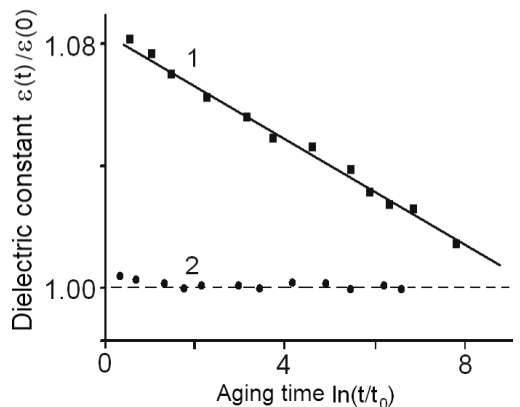

Fig. 4. Relative dielectric constant versus logarithm of aging time $t / t_{0}\left(t_{0}=1 \mathrm{~min}\right)$ after removing uniaxial compression 300 bar. 1 FE solid solution PZT 52/48, 2 - AFE solid solution PZT 98/02.

Of all the types of actions to test our hypothesis, uniaxial stress is the most suitable. In this case, there is no charge from external sources, and we use weak stress intensity that does not lead to a substantial change of crystal and domain structures.

Figure 4 shows the relaxation dependences for solid solutions PZT 98/2 and 52/48 after being subjected to uniaxial compression. The first of the said solid solutions is an antiferroelectric, and the second is ferroelectric. As can be seen, for AFE solid solution the relaxation virtually does not exist.

The following section of this paper represents the results of experiments made for testing our hypothesis about the mechanism of long-time relaxation.

\section{Nonreversible Relaxation After Thermal Action}

This section presents the results of the investigation of nonlinear relaxation characteristics and their connection with structure variations of piezoelectric ceramics that are caused by sample annealing. Now annealing temperature interval extends up to Curie point. $\left(\mathrm{Pb}_{0.95} \mathrm{Sr}_{0.05}\right)\left(\mathrm{Zr}_{1-x} \mathrm{Ti}_{x}\right) \mathrm{O}_{3}$-based solid solutions with $0.40 \leq x \leq 0.60$ (both hard- and soft ferroelectric ceramics) were taken as study objects. For boundary zirconium content, solid solutions are single-phase in the crystal structure sense with $T$ ( $x$-value is in the vicinity of 0.60$)$ or $R h$ ( $x$-value is in the vicinity of 0.40) type of lattice distortions. Solid solutions with $\mathrm{Zr} / \mathrm{Ti}$ content in the vicinity of 52/48 are placed in the morphotropic region of the PZT phase diagram $-T$ - and $R h$-distorted domains coexist in the volume of solid solutions.

\subsection{Experimental results}

Figure 1 shows time dependences of the dielectric constant for the ceramic sample of $\left(\mathrm{Pb}_{0.95} \mathrm{Sr}_{0.05}\right)\left(\mathrm{Zr}_{0.53} \mathrm{Ti}_{0.47}\right) \mathrm{O}_{3}$ solid solution after annealing at different temperatures and quick cooling (these dependences are obtained for polarized sample). Now we will consider wider interval of annealing temperatures than one in Sec. 3 of the present paper.
As it is shown in Fig. 1, we can distinguish the characteristic temperature $T_{d}=240^{\circ} \mathrm{C}$, and the temperature interval of the sample annealing before quenching can be divided into two parts: $T_{\text {ann }}<T_{d}$ and $T_{\text {ann }}>T_{d}$. Temperature $T_{d}$ is characterized by two distinctive features.

First of all, the dielectric constant after annealing at temperatures $T_{\mathrm{ann}}<T_{d}$ increases (temperature interval $\mathrm{I}$ in Fig. 1), while it decreases after annealing at temperatures above $T_{d}$ (temperature interval II).

Secondly, if $T_{\mathrm{ann}}<T_{d}$, the changes in the sample properties are reversible in the process of heating and subsequent aging. In the case of $T_{\text {ann }}>T_{d}$, irreversible change in the sample properties takes place.

The dependences of the coefficients $A$ and $B$ on annealing temperature have nonlinear character and are characterized by the maxima at $T_{\mathrm{ann}}=T_{d}$ (Fig. 5). This figure shows the dependence of the relaxation time $\tau$ on the annealing temperature $T_{\text {ann }}$ also. As seen from these dependences, temperature $T_{d}$ is characterized by the maximum of the relaxation rate.

Temperature dependences of the dielectric constant and remnant polarization were measured for revealing the physical nature of the characteristic temperature $T_{d}$. Figure 6 shows the temperature dependence of the dielectric constant,
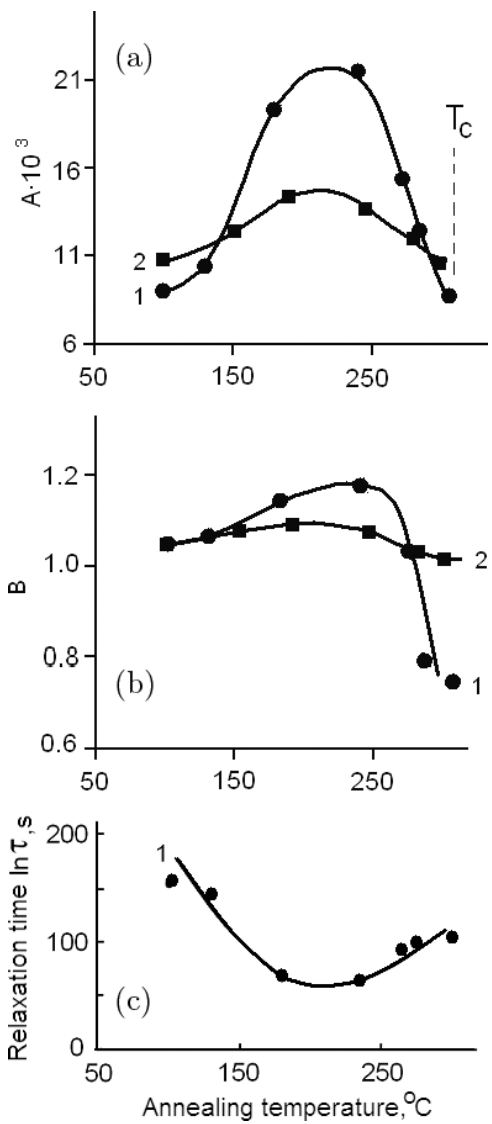

Fig. 5. Coefficients $A$ and $B$, defined by Eq. (1), and relaxation time $\tau$ versus annealing temperature for polarized (1) and depolarized (2) samples of hard $\left(\mathrm{Pb}_{0.95} \mathrm{Sr}_{0.05}\right)\left(\mathrm{Zr}_{0.53} \mathrm{Ti}_{0.47}\right) \mathrm{O}_{3}$-based ferroelectric ceramic. 


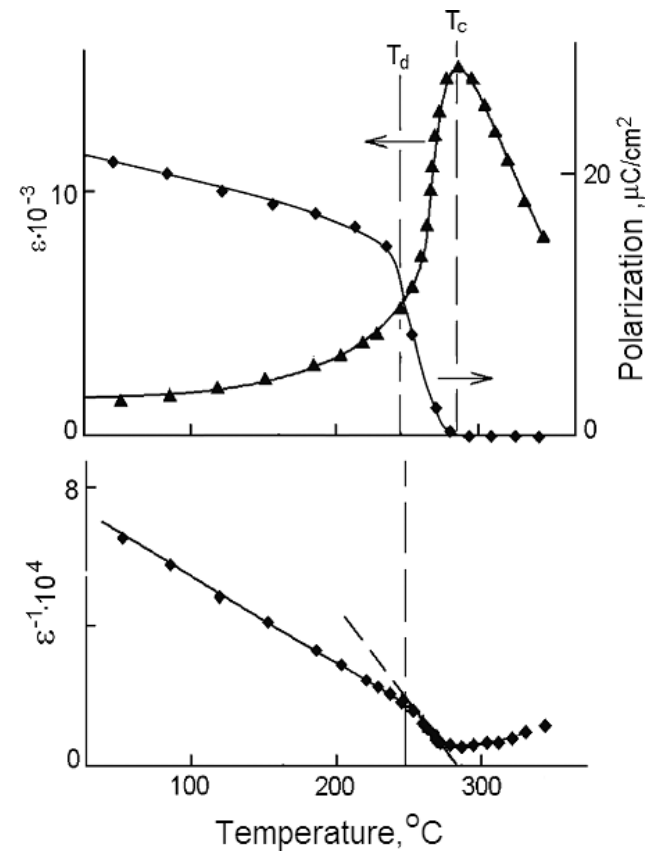

Fig. 6. Dielectric constant, reciprocal dielectric constant and remnant polarization versus temperature for $\left(\mathrm{Pb}_{0.95} \mathrm{Sr}_{0.05}\right)\left(\mathrm{Zr}_{0.53} \mathrm{Ti}_{0.47}\right) \mathrm{O}_{3}$ solid solution.

remnant polarization and the inverse dielectric constant. In aggregate, these dependencies clear up completely the nature of the temperature $T_{d}$. Despite the fact that the temperature $T_{d}$ is below the Curie point $T_{C}$ of the investigated solid solution, the domain structure destruction of the sample begins in the vicinity of temperature $T=T_{d}$. At the same time, the maximum of the temperature dependence $\varepsilon(t)$ corresponds to the Curie point $T_{C}$. At the point $T_{d}$, the temperature dependence of the inverse dielectric constant deviates from the classical linear law.

Temperature $T_{d}$ separates the temperature ranges of reversible and irreversible changes in the dielectric constant (as consequence, in remnant polarization also). The initiation of partial domain structure disordering at the temperature $T_{\mathrm{ann}}=$ $T_{d}$ is confirmed by the variation in the profile of X-ray lines on the X-ray patterns that occurs after annealing at temperatures $T_{d}<T_{\text {ann }}<T_{c}$ (Fig. 7). Analysis of diffraction patterns showed that for the samples annealed at a temperature $T_{d}<T_{\text {ann }}<T_{C}$, there is a significant redistribution of the intensities of the doublet lines which testifies to the domain disordering. For $T_{\text {ann }}<T_{d}$, the shape of X-ray lines does not only depend on the annealing temperature and coincides with the one of polarized samples at room temperature.

For both partially and fully depolarized samples the dependence of $A$-parameter on the annealing temperature has a maximum. Its height decreases with increasing depolarization degree of the sample, and the whole curve $A\left(T_{\text {ann }}\right)$ for depolarized sample lies below the corresponding curve for polarized one. Corresponding curves are shown in Fig. 5.

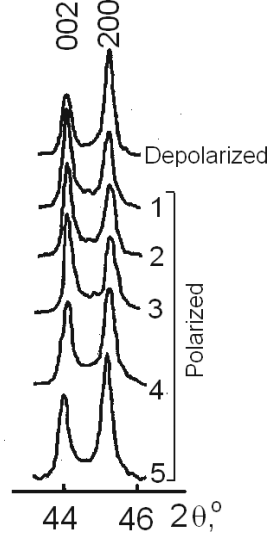

(a)

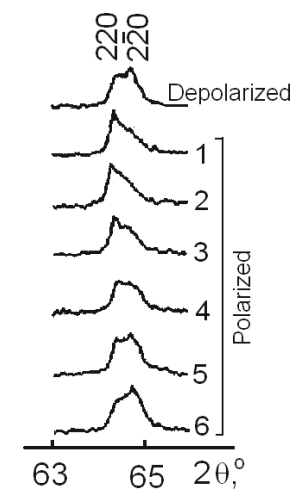

(b)
Fig. 7. Change of the shape of X-ray line (200) of $\left(\mathrm{Pb}_{0.95} \mathrm{Sr}_{0.05}\right)$ $\left(\mathrm{Zr}_{0.46} \mathrm{Ti}_{0.54}\right) \mathrm{O}_{3}$ solid solution (a) and (220) line of $\left(\mathrm{Pb}_{0.95} \mathrm{Sr}_{0.05}\right)$ $\left(\mathrm{Zr}_{0.58} \mathrm{Ti}_{0.42}\right) \mathrm{O}_{3}$ solid solution (b) after annealing at different temperatures. (a) $T_{c}=338^{\circ} \mathrm{C}, T_{d}=299^{\circ} \mathrm{C} ; T_{\text {ann }},{ }^{\circ} \mathrm{C}: 1-151,2-$ 173, $3-275,4-309,5-328$. (b) $T_{c}=296^{\circ} \mathrm{C}, T_{d}=277^{\circ} \mathrm{C}$; $T_{\mathrm{ann}},{ }^{\circ} \mathrm{C}: 1-173,2-214,3-259,4-278,5-285,6-293$.

This indicates that the relaxation rate of depolarized sample is lower than for the polarized one.

Similar results were obtained in the study of long-term relaxation in ferro-hard and ferro-soft solid solutions (with identical content of $\mathrm{Zt}$ and Ti). For both cases, dependences of $A$-parameter on the annealing temperature are identical and have extreme at the critical temperature $T_{d}$ (Fig. 8). However, the relaxation rate for hard sample is significantly higher. Some difference of $T_{d}$ for hard and soft ceramics is caused by different doping elements in solid solutions (more on this will be somewhat below).

If the sample's face ends were in short-circuited state at annealing, the relaxation rate is lower, then without short circuit.

The results obtained in the study of long-term relaxation after complex action on the sample - DC electric field and heating - are shown in Fig. 9. Electric field does not lead to a change of the nature of $A\left(T_{\mathrm{ann}}\right)$ and $\tau\left(T_{\mathrm{ann}}\right)$ curves.

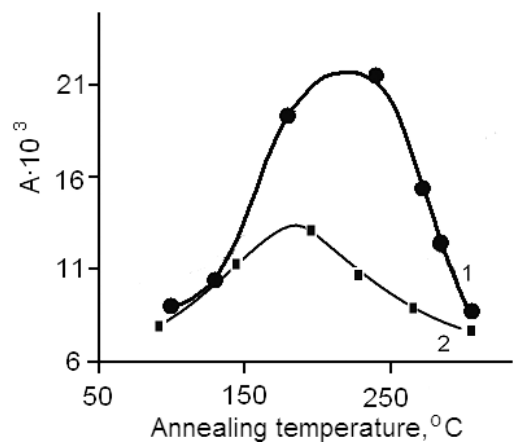

Fig. 8. A-coefficient versus annealing temperature for hard (1) and soft (2) ceramics on the base of $\left(\mathrm{Pb}_{0.95} \mathrm{Sr}_{0.05}\right)\left(\mathrm{Zr}_{0.53} \mathrm{Ti}_{0.47}\right) \mathrm{O}_{3}$ solid solution. 

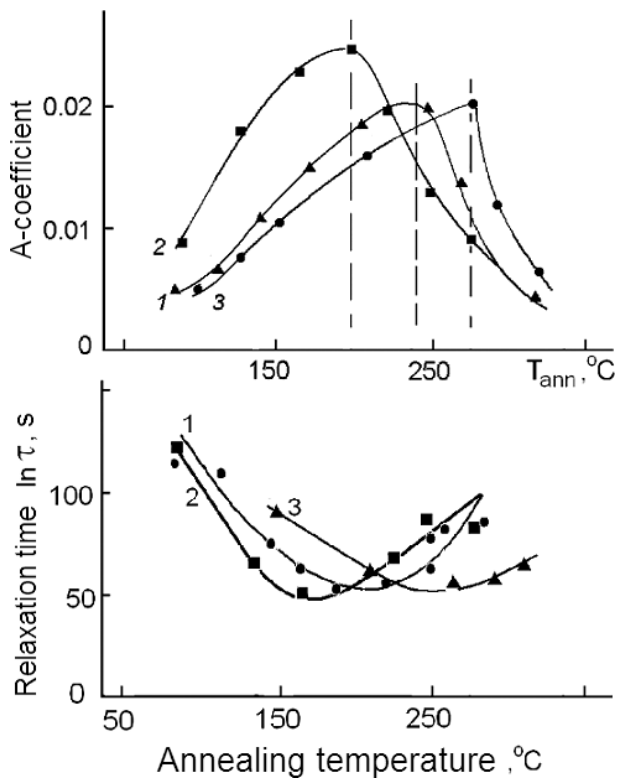

Fig. 9. A-coefficient and relaxation time $\tau$ versus the temperature of annealing without field (1) and under the action of DC field with intensity $300 \mathrm{~V} / \mathrm{mm}$ directed along (3) and against (2) the sample's polarization.

However, the temperature $T_{d}$ depends on the field direction. When the field and sample's polarization are directed against one to an other, $A\left(T_{\text {ann }}\right)$ and $\tau\left(T_{\text {ann }}\right)$ shift to lower temperatures. When the field direction coincides with the direction of polarization, these dependences are shifted to the high temperature region (Fig. 9). The dependence of the critical temperature $T_{d}$ on the field intensity is practically linear for weak fields (until $P(E)$-dependence is a linear in the hysteretic loop and a partial depolarization of the sample does not take place).

Annealing temperature $T_{d}$ defines the beginning of the domain structure reconstruction, i.e., a partial depolarization of the sample. DC electric field increases the stability of a single-domain structure at the coincidence of its and the polarization directions and reduces the stability of this structure for the opposite direction of the external field. The last case in our experiments in own nature is close to that happening near the coercive field at the sample repolarization by electric field.

The study of the dielectric constant relaxation in the solid solutions from the $T$ and $R h$ regions of the PZT phase diagram gives another result in favor of our hypothesis about the mechanism of long-term after-effect relaxation. In $T$ solid solutions in the polarized state the dielectric constant is larger than in the depolarized one. During thermal depolarization, dielectric constant is reduced (Fig. 10). In the $R h$ solid solutions all are on the contrary. In the polarized state, the dielectric constant is lower than that in the depolarized one, and it increases at the depolarization process. However, during after-annealing relaxation the dielectric constant in both cases only decreases and never increases.

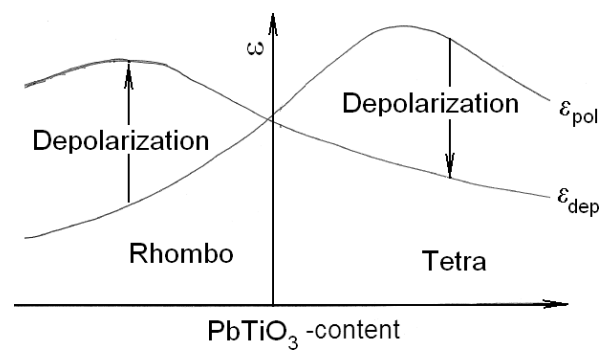

Fig. 10. Schematic behavior of dielectric constant at the depolarization process for solid solutions from $R h$ and $T$ region of the PZT phase diagram.

With the growth of the $\mathrm{Zr}$-content (at the passage from $T$ into $R h$-region of the phase diagram) the temperature interval $\Delta=T_{C}-T_{d}$ decreases (from $45^{\circ} \mathrm{C}$ at $\mathrm{Zr}$-content of $40 \%$ to $15^{\circ} \mathrm{C}$ at $\mathrm{Zr}$-content of $60 \%$ ). The reason for $T_{d}$ keeping away from $T_{C}$ for $T$ solid solutions is the higher value of the reduced deformation parameter $\delta$ for $T$-phase as compared with the $R h$-phase $(\delta \approx \cos \alpha$ for $R h$-phase and $\delta \approx(2 / 3) \times$ $[(c / a)-1]$ for $T$-phase), resulting in greater elastic energy of dipole ordered state for $T$-phase. Additionally, $90^{\circ}$-domains give contribution to sample's elastic energy also. At heating, greater elastic energy leads to an earlier (relative to Curie point) destruction of the dipole ordered state.

\subsection{Discussion and confirmation of the hypothesis of long-term relaxation}

The explanation of all the above demonstrated experimental results can be given only on the basis of the hypothesis proposed in Sec. 3.2 that takes into account the oxygen vacancies in the solid solutions and $F^{+}$- and $F^{0}$-center creation.

The increase in the relaxation rate with increasing annealing temperature in the temperature range $T<T_{d}$ is caused by the fact that an increase in temperature leads to a greater decrease of the remnant polarization of the sample and, accordingly, to the greater value the released charge (electrons). $F^{0}$-center energy is greater then the energy of $F^{+}$-center, so their numbers on the end surfaces of the sample is less and their contribution to the relaxation process is weaker than the contribution of $F^{+}$-centers. When $T_{\text {ann }}$ increases, realized charge increases and the number of $F^{0}$-center increases also. Thus, their contribution to the electric field, induced by charged $F$-centers, increases. Since $F^{0}$-centers are energetically less stable than the $F^{+}$-centers, they relax into the oxygen vacancies $\left(F^{0} \rightarrow V_{O}^{\bullet \bullet}\right)$ faster. For this reason, induced electric field diminishes with larger rate. Therefore, an increase in annealing temperature (for $T_{\text {ann }}<$ $T_{d}$ ) results in the increase in the relaxation rate - it leads to an increase in the coefficient $A$ and decrease in the relaxation time $\tau$ in Fig. 5.

When annealing temperatures exceed $T_{d}\left(T_{\mathrm{ann}}>T_{d}\right)$, a partial destruction of domain ordering occurs in polarized sample (there is a turn of directions of the spontaneous 
polarization of each domain from the polarization direction of the sample as a whole, and remnant polarization $P_{r}$ decreases). For this reason, less electric charge is realized on the end faces of the sample during annealing. Naturally, the amount of charged $F^{0}$-centers is reduced relative on the number of $F^{+}$-centers. All this leads to a decrease of the relaxation rate at the annealing temperature at $T_{\text {ann }}>T_{d}$.

In partially depolarized samples, less spontaneous polarization domains locate along the normal to the end surfaces of the sample than in the polarized one. Upon annealing, less charge is allocated on these surfaces, less charged $F^{+}$- and $F^{0}$-centers are formed on these surfaces of the quenched samples. Accordingly, the electric field, which is created by these defects of the crystal lattice, is smaller. Consequently, in depolarized samples, relaxation rate is lower and the curve $A\left(T_{\text {ann }}\right)$ lies below (see Fig. 5) than corresponding curve in the polarized sample.

The difference of relaxation rate in hard and soft solid solutions (see Fig. 8) finds its explanation in the framework of the hypothesis proposed in Sec. 3.2 also. Hard or soft ferroelectric ceramics may be obtained on the base of the same PZT solid solution, at the same ratio of $\mathrm{Zr} / \mathrm{Ti}$. In the former case, solid solution is doped by monovalent ions, substituted lead in the $A$-sublattice of the crystal structure, or trivalent ions substituted $B$-ions of parent solid solution. ${ }^{25}$ For obtaining soft PZT-based ceramics, lead is substituted by trivalent ion or in the way of the substitution of $B$-ions by pentavalent ions. ${ }^{25}$ As a result of compliance with the rule of electrical neutrality, hard ferroelectric ceramics contain more oxygen vacancies. In the study of the present paper, the piezoelectric soft solid solutions contain, the trivalent bismuth as the doping element (for lead substitution) and ferroelectric hard ceramics contain the trivalent manganese ions into $B$-sites of the crystal lattice (as the doping element too).

At heating, the above discussed mechanism of electron capture by oxygen vacancies takes effect. The subsequent relaxation of dielectric constant after cooling samples is a consequence of the relaxation of these centers to the ground state ("pure" oxygen vacancy) and depression to zero the field formed by the said charged defects. More oxygen vacancies in hard ferroelectric ceramics provide larger amount of $F^{0}$-centers, and as a result, larger relaxation rate in the aging process.

The results obtained in the study of long-term relaxation after complex external effect of DC electric field and heating are in full compliance with the proposed hypothesis also. The role of external electric field is reduced only to the fact that it improves the stability of the domain ordered state when its direction coincides with the direction of the sample's polarization, and reduces the stability of this state for opposite direction of the field. Therefore, in the first case the electric field increases the temperature $T_{d}$, and in the second case the field reduces this temperature (see Fig. 9).

The results obtained in the study of relaxation processes in solid solutions from various regions of PZT phase diagrams $-R h$ and $T-$ are also fully consistent with the hypothesis about the mechanism of the after-effect relaxation considered in Sec. 3.2. Moreover, they can find their consistent explanation only in the context of this hypothesis.

As noted above, during the process of partial heating depolarization (annealing temperature range is $T_{d}<T_{\text {ann }}<$ $T_{C}$ ) in solid solutions from the $T$-region of the phase diagram, the dielectric constant decreases, while in solid solution from the $R h$-region it increases (see Fig. 10). After partial depolarization and cooling to room temperature, in the process of the relaxation the dielectric constant only decreases in both cases. Such behavior of the dielectric constant is a consequence of the fact that in both $T$ and $R h$ solid solutions (regardless of the type of distortion of the crystal lattice) during annealing, uncompensated charge is released and the formation of $F$-centers take place. In both cases after cooling, samples are under the influence of induced electric field that promotes to increasing the relative permittivity. In the process of relaxation, field strength decreases, the value of the dielectric constant also decreases.

\section{Conclusions}

This paper presents the results of the study of relaxation processes in piezoelectric PZT-based solid solutions after external affects the sample. The larger part of the paper considers thermal action and relaxation after rapid sample cooling.

After weak external effects that do not lead to a rearrangement of the domain structure, the relaxation is reversible. The dependence of the relaxation rate on the action intensity has linear character.

After thermal treatment leading to a partial rearrangement of the domain structure, relaxation is irreversible - after thermal action termination the dielectric constant does not return to its initial value. In this case, the dependence of the relaxation rate on the annealing temperature is nonlinear there is a critical value $T_{d}$ of annealing temperature at which the relaxation rate is maximum. In the vicinity of the said critical annealing temperature, the domain structure reorientation begins.

As a result of the experimental data analysis, at first a hypothesis was put forward about the nature of long-term relaxation in ferroelectric PZT-based solid solutions. In the process of the work, this hypothesis is turned into a physical model, having the right to exist. The model takes into account the presence move off. Both the remnant polarization decrease and free charge (unbound by polarization) realization at the surface ends of the sample (or domains) take place during the external actions. This charge is captured by oxygen vacancies, and $F^{+}$- and $F^{0}$-centers are formed. $F$-centers induce an electric field inside the sample that results in deviation of dielectric constant from the initial value.

This field is retained after removal of the external influences on the sample during the lifetime of the charged 
$F$-centers. At the $F$-centers decay $\left(F^{0}, F^{+} \rightarrow V_{\ddot{O}}\right)$, electric field created by them decreases to zero. During the $F$-centers decay dielectric constant decreases to its initial value also.

Most of the results presented in this paper are connected with the restructuring of the domain structure. However, no domain restructuring determines the mechanism of aftereffect long-term relaxation. The relaxation of dielectric constant is determined by the behavior of crystal lattice oxygen vacancies. Rearrangement of the domain structure determines peculiarities of the relaxation process only, in particular, the nonlinear dependence of the relaxation rate on the annealing temperature, but not its own relaxation mechanism.

This paper presents results of the study of the after-thermal relaxation. These results confirm the model of long-term relaxation based on $F$-center formation and subsequently their decay. The most favorable results are obtained after thermal treatment leading to the domain structure disordering (full or partial). Results obtained after action of DC electric field and uniaxial pressure onto samples acknowledge the said model also. As in the case of thermal action, the most interesting results are obtained after effects which degrade the domain structure of polarized samples. These results will be presented in the next paper.

\section{References}

${ }^{1}$ C. Burfoot and G. W. Taylor, Polar Dielectrics and Their Applications (The McMillan Press LTD, Great Britain, 1979).

${ }^{2}$ Y. Xu, Ferroelectric Materials and Their Applications (NorthHolland, Amsterdam, 1991).

${ }^{3}$ J. Yang, Analysis of Piezoelectric Devices (University of Nebraska-Lincoln, USA, 2006).

${ }^{4}$ C.-C. Wu, Cheng-Chun Lee, G. Z. Cao and I. Y. Shen, Effects of corner frequency on bandwidth and resonans amplitude in designing PZT thin-film actuators, Sens Actuators A 125, 178 (2006).

${ }^{5}$ C. S. Ganpule et al., Polarization relaxation kinetics and $180^{\circ}$ domain wall dynamics in ferroelectric thin films, Phys. Rev. B 65 , 014101 (2001).

${ }^{6}$ D. Zhoua and M. Kamlah, Determination of room-temperature creep of soft lead zirconate-titanate piezoelectric ceramics under static electric fields, J. Appl. Phys. 98, 104107 (2005).

${ }^{7}$ E. M. Bourim et al., Creep behavior of undoped and $\mathrm{La}-\mathrm{Nb}$ codoped PZT based micro-piezoactuators for micro-optical modulator applications, Sens. Actuators A 155, 290 (2009).

${ }^{8}$ S.-J. Kima, J. H. Kima and C.-H. Lee, Domain switching and creep behavior of a poled PZT wafer under through thickness electric fields at high temperatures, Acta. Mater. 58, 2237 (2010).
${ }^{9}$ S. A. Gridnev, V. M. Popov and L. A. Shuvalov, The processes of slow relaxation in TGS monocrystals, Izv. Akad. Nauk. SSSR: Ser. fiz. 48, 1226 (1984) (in Russian).

${ }^{10}$ R. Street, J. C. Woolley and P. B. Smith, Magnetic viscosity under discontinuously and continuously variable field conditions, Proc. Phys. Soc. B 65, 679 (1952).

${ }^{11}$ S. Krupička, Physik Der Ferrite (Academia, Prague, 1973).

${ }^{12}$ D. V. Kuzenko, V. M. Ishchuk, A. I. Bazhin and N. A. Spiridonov, After-effect relaxation process in lead zirconatr-titanate, Solid State Phys. 54, 896 (2012) (in Russian).

${ }^{13}$ L. Hong, A. K. Soh, Q. G. Du and J. Y. Li, Interaction of Ovacancies and domain structures in single crystal $\mathrm{BaTiO}_{3}$ : Twodimensional ferroelectric model, Phys. Rev. B 77, 094104 (2008).

${ }^{14}$ C. H. Park and D. J. Chadi, Microscopic study of oxygen-vacancy defects in ferroelectric perovskites, Phys. Rev. B 57, R13961 (1998).

${ }^{15}$ A. Stashans, S. Serrano and P. Medina, A quantum-chemical study of oxygen-vacancy defects in PbTiO3 crystals, Physica B 381, 82 (2006).

${ }^{16}$ E. Cockayne and B. P. Burton, Dipole moment of a Pb-O vacancy pair in $\mathrm{PbTiO}_{3}$, Phys. Rev. B 69, 144116 (2004).

${ }^{17}$ W. J. Merz, Domain formation and domain wall motions in ferroelectric $\mathrm{BaTiO}_{3}$ single crytals, Phys. Rev. 95, 690 (1954).

${ }^{18} \mathrm{~K}$. Amanuma, T. Hase and Y. Miyasaka, Fatigue Characteristics of Sol-Gel Derived $\mathrm{Pb}(\mathrm{Zr}, \mathrm{Ti}) \mathrm{O}_{3}$ Thin Films, Jpn. J. Appl. Phys. Part 1 33, 5211 (1994).

${ }^{19}$ W. H. Shepherd, in Ferroelectric Thin Films, eds. E. R. Myers and A. I. Kingon, MRS Symposia Proceedings Series, Vol. 200 (Materials Research Society, Pittsburgh, 1990), p. 277.

${ }^{20}$ E. A. Kotomin, R. I. Eglitis and G. Borstel, Quantum chemical modelling of point defects in $\mathrm{KNbO}_{3}$ perovskite crystals, Comput. Mater. Sci. 17, 290 (2000).

${ }^{21}$ C. Duque and A. Stashans, Structural and optical properties of $F$-center defects on barium titanate $<001>$ surface, Mater. Lett. 57, 3954 (2003).

${ }^{22}$ R. I. Eglitis, $A b$ initio calculations of $\mathrm{SrTiO}_{3}, \mathrm{BaTiO}_{3}, \mathrm{PbTiO}_{3}$, $\mathrm{CaTiO}_{3}, \mathrm{SrZrO}_{3}, \mathrm{PbZrO}_{3}$ and $\mathrm{BaZrO}_{3}$ (001), (011) and (111) surfaces as well as F-centers, polarons, KTN solid solutions and $\mathrm{Nb}$ impurities therein, Intern. J. Mod. Phys. B 28, 1430009 (2014).

${ }^{23} \mathrm{~S}$. Teslic and T. Egami, Atomic structure of $\mathrm{PbZrO}_{3}$ determined by pulsed neutron diffraction, Acta Cryst. B 54, 750 (1998).

${ }^{24}$ G. Kugel, I. Jankowska-Sumara, K. Roleder and J. Dec, High temperature Raman light scattering in $\mathrm{PbZrO}_{3}$ single crystals, J. Korean Phys. Soc. 32, S581 (1998).

${ }^{25}$ B. Jaffe, W. R. Cook and H. Jaffe, Piezoelectric Ceramics (Academic Press, London and New York, 1971). 